\title{
Rock Art Interpretation within Indiana MAS
}

\author{
Viviana Mascardi ${ }^{1}$, Vincenzo Deufemia ${ }^{2}$, Damiano Malafronte ${ }^{1}$, \\ Alessandro Ricciarelli ${ }^{1}$, Nicoletta Bianchi ${ }^{3}$, Henry de Lumley ${ }^{3}$ \\ 1 Università di Genova \\ Via Dodecaneso 35, 16146, Genova, ITALY \\ viviana.mascardi@unige.it, damianomal@gmail.com, \\ ricciarelli.alessandro@gmail.com \\ 2 Università di Salerno \\ Via Ponte don Melillo, 84084 Fisciano (SA), ITALY \\ deufemia@unisa.it \\ ${ }^{3}$ Lab. Dép. de Préhistoire du Lazaret \\ 33 Bis Boulevard Franck Pilatte, 06300 Nice, FRANCE \\ \{nbianchi, lazaret\}@lazaret.unice.fr
}

\begin{abstract}
This paper presents the first results achieved within the Indiana MAS project funded by Italian Ministry for Education, University and Research, MIUR. We discuss how the AgentSketch holon belonging to the Indiana MAS has been extended to cope with images, besides hand drawn sketches, and has been tested in the domain of Mount Bego's prehistoric rock art (southern French Alps). The way Indiana MAS holons cooperate in order to provide correct interpretations of ambiguous shapes is discussed by means of an example based on hypotheses recently advanced by archaeologists.
\end{abstract}

\section{Introduction}

This paper presents the first results achieved within the project "Indiana MAS and the Digital Preservation of Rock Carvings: A Multi-Agent System for Drawing and Natural Language Understanding Aimed at Preserving Rock Carvings", funded by Italian Ministry for Education, University and Research, MIUR. The project, accepted for funding in September 2011, will start in March 2012.

The aim of the project is to develop a technology platform based on intelligent software agents for the digital preservation of rock carvings, which both integrates and complements the techniques usually adopted to preserve heritage sites. The platform will support domain experts in the creation of a repository, which may become a reference at Italian and European level as a thorough database of rock carvings, and in the interpretation of rock carvings. It will also promote the awareness and the preservation of the cultural treasure by making cultural information accessible to all on the Internet and preserve it for future generations. To this end, the Indiana MAS platform will enable the preservation of all kinds of available data about rock carvings, such as images, geographical objects, textual descriptions of the represented subjects. It will provide the 
means to organize and structure such data into an existing collaborative tool set, and will supply domain experts with collaborative facilities for processing data and making assumptions about the way of life of the ancient people based on them.

The choice of agent technology for addressing Indiana MAS goals was a very natural one, given the need that each component of the system, while operating in a highly autonomous way, interacts and coordinates with the other components to share information and to reason about them in the most effective way.

The paper is organized in the following way: Section 2 introduces the Indiana MAS architecture and provides some background information on AgentSketch. Section 3 discusses how the AgentSketch component of Indiana MAS has been extended to cope with images, besides hand drawn sketches, and has been tested in the domain of prehistoric rock art, and illustrates how correct interpretation can result from interaction between AgentSketch and other MAS components. Section 4 concludes and outlines our future work.

\section{Indiana MAS and AgentSketch}

Indiana MAS integrates intelligent software agents, ontologies, natural language processing and sketch recognition techniques. Multi-agent systems (MASs) represent an optimal solution to manage and organize data from multiple sources and to orchestrate the interaction among the components devoted to the interpretation of the carvings. Ontologies allow to define a common vocabulary that can be profitably exploited to organize data associated with rock carvings, included their semantic annotations, and create semantic relationships between them. Natural Language Processing techniques are used to extract relevant concepts from text and for mining semantic relationships among them, hence supporting the definition and evolution of ontologies devoted to describe the domain. Sketch recognition techniques are applied to classify the elementary shapes of the carving drawings and associate their possible interpretations with them.

Fig. 1 describes the Indiana MAS holonic architecture. Each component represented by a 3D puppet in the figure is a holon: it consists of "agents that give up parts of their autonomy and merge into a super-agent (a holon), that acts when seen from the outside - just as a single agent again" [8].

Starting from structured and unstructured multilingual and multimedia data coming from different sources (the Bicknell Legacy owned by the University of Genova including images of Bicknell's tracings with metadata in English and in Italian; the ADEVREPAM database owned by the Laboratoire Départementale de Préhistoire du Lazaret storing images of the tracings made by De Lumley's team with metadata in English and French; heterogeneous data coming from other open sources such as the World Wide Web), we created a first repository of raw data. 


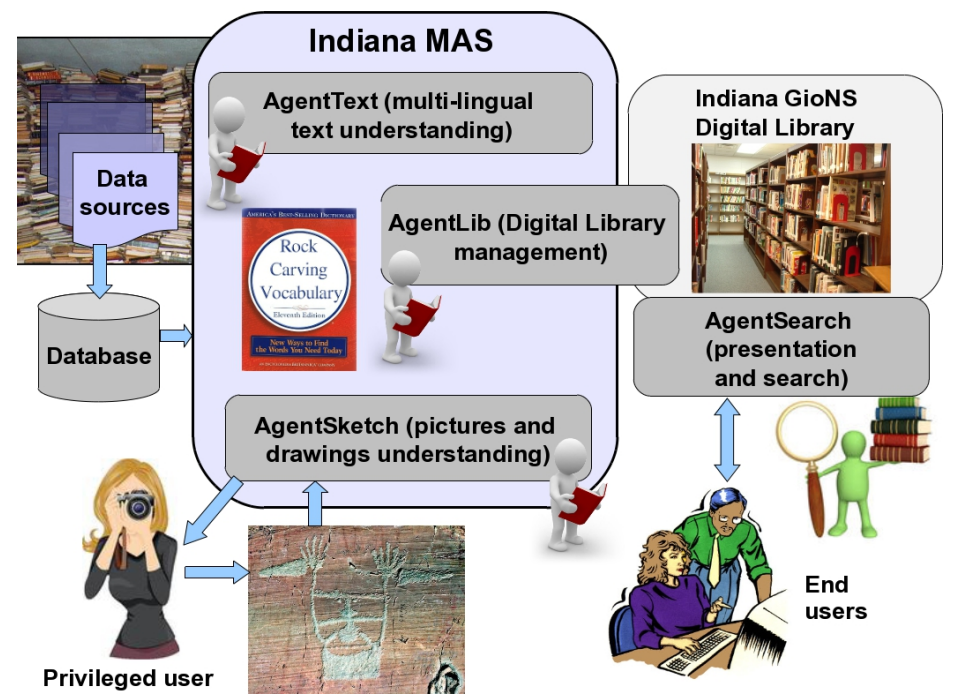

Fig. 1. Indiana MAS architecture

AgentText takes care of understanding text in English, French, and Italian, and classifying it according to the Rock Carving Vocabulary that dynamically evolves as new data will be inserted into the repository.

Image and drawing understanding are performed by AgentSketch [2], a multiagent system that we developed as part of our previous research, and that will be discussed in the sequel. Classified data (text, images, drawings) are made available to the public thanks to the Indiana GioNS Digital Library. The Library is managed by AgentLib, that interacts with both AgentText and AgentSketch to help relationships among heterogeneous data emerge and be stored into the Digital Library as new knowledge. The Library is made accessible to users by AgentSearch, a personal user assistant designed along the lines of old and wellknow "digital butlers" $[4,10]$, providing user profiling and content personalization capabilities. The interface offered by AgentSearch integrates query-by-sketch and natural language understanding technologies.

While the access point to Indiana MAS services for end users is AgentSearch, each holon in the MAS can be directly accessed by privileged users, as shown in Fig. 1 where an archaeologist inputs the image of Mount Bego's most famous engraving, the "wizard", to AgentSketch directly, for directly obtaining an interpretation of the image.

AgentSketch is composed by the four kinds of agents shown in Fig. 2.

Interface Agent. It represents an interface between the agent-based AgentSketch framework and the generic "Input Suppliers" that are not included inside the framework. The nature of these input suppliers may vary according to the type of sketch to be interpreted and to the drawing process (on-line vs. off-line). 


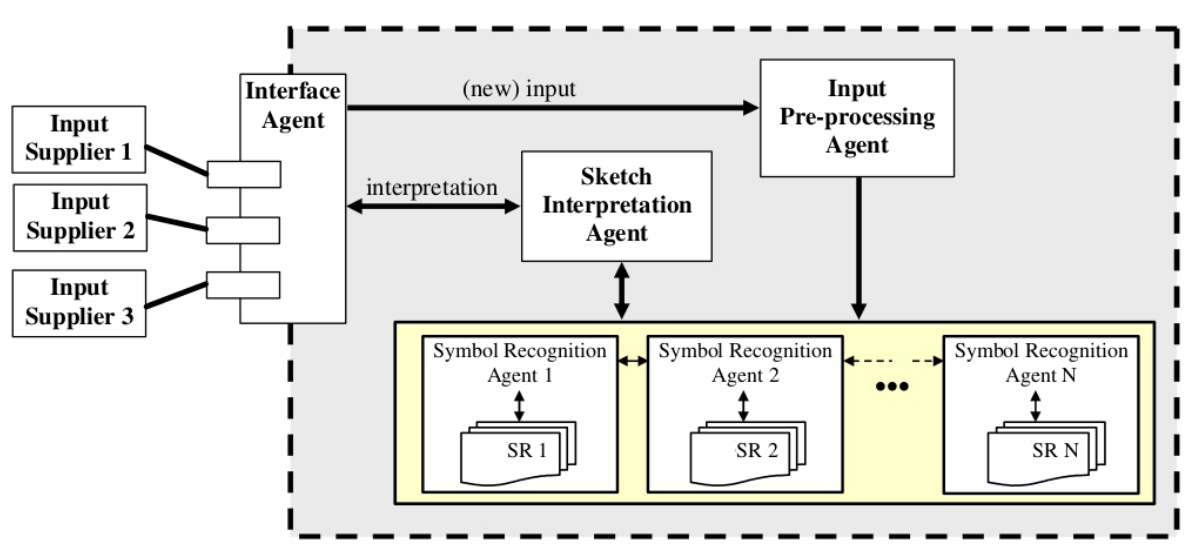

Fig. 2. AgentSketch architecture

The Interface Agent informs the "Sketch Interpretation Agent" (SIA) and the "Input Pre-Processing Agent" (that, in turns, informs the "Symbol Recognition Agents") about the nature of the recognition process (off-line or on-line) and converts the information produced by the input suppliers into a suitable format for these agents. It sends each new available piece of input (or the whole input, in case of an off-line recognition process of an image) to the Input Pre-Processing Agent, and interacts with the SIA for sending the sketch interpretation requests to it, and for delivering its answer to the user. For example, the implemented instance of the Interface Agent currently available within AgentSketch provides a stub for an on-line editor that takes advantage of Java Swing components and Satin, and for a file browser allowing the user to select images from the file system.

Input pre-processing agent. It processes the input received from the Interface Agent and sends the obtained results to the "Symbol Recognition Agents" described in the following, using a format compliant with the recognition approach they apply.

Symbol Recognition Agents (SRAs). Each SRA is devoted to recognize a particular symbol of the domain. Moreover SRAs may collaborate with other SRAs in order to apply context knowledge to the symbols they are recognizing, and with the SIA that deals with the sketch interpretation activity. On-line recognition of hand-drawn sketches and off-line recognition of objects within images are based on very different approaches, and exploit different algorithm and existing libraries to work. This lower level activity is demanded to "Symbol Recognizers" (SRs in the figure) that are not agents, lacking most of the agentcharacterizing features, but just software modules managed by SRAs. As long as there is one SRA that correctly integrates SRs by managing their execution as well as data conversion issues, the actual implementation of the SRs and the approach to recognition that they adopt do not matter. The inherently flexible 
and modular agent-based approach allowed us to seamlessly cope with symbols that have been recognized by heterogeneous SRs managed by ad-hoc SRAs.

Sketch Interpretation Agent (SIA). The SIA provides the correct interpretation either of the sketch drawn so far (in case of an on-line drawing process) or of the entire sketch (in case of an off-line recognition process) to the Interface Agent. In particular, it analyzes the information received from SRAs and solves conflicts between symbols that might arise. When all the conflicts have been solved, the SIA proposes the sketch interpretation to the user, interacting with the Interface Agent.

AgentSketch has been experimented in the domain of Use Case Diagrams [2]. Its exploitation for reasoning about hand-drawn sketches in the physical security domain has been discussed in [3]. In the following section we discuss two SRAs operating in the rock art domain, and the interactions among AgentSketch and the other holons in the Indiana MAS to reach an agreement on the correct interpretation of a sketch, based on external information coming from heterogeneous and multi-lingual documents.

\section{Recognizing and Interpreting Rock Carvings in Indiana MAS}

\subsection{SRA for Sketches}

In order to recognize rock art carvings while they are being sketched, we implemented a SRA working on-line and based on LADDER [9]. In LADDER, symbol recognition is performed using the rule-based system Jess [7]. In particular, for each symbol of the domain, a Jess rule is automatically generated from a LADDER structural shape description, which mainly contains information on the shape of the symbol. Recognition using Jess is sensible neither to the order of the strokes, nor to the symbol dimension. These features make the approach very stable.

As an example, the LADDER description of a corniculate is shown in Fig. 3. This rule defines a corniculate as one ellipse and four lines from which the shape is built, plus the topological constraints defining the relationships among these elements: two lines (representing the lower part of the horns) must be similar in length, touch the ellipse (representing the head), and be over it; the two other lines (representing the upper part of the horns) must be similar in length, and each of them must be above the lower part of one horn and connected to it.

The final behavior of this rule is that, when in the working memory of the LADDER application there are four lines and one ellipse that respect the precondition of the rule, the rule is fired and a corniculate symbol is recognized. A similar (but simpler) rule has been defined for recognizing short weapons.

The shapes described with LADDER must be diagrammatic or iconic since they have to be drawn using a predefined set of primitive shapes and composed using a predefined set of constraints.

Fig. 4 shows a screenshot of AgentSketch at work. Fig. 4(a) shows the symbols sketched by the user, and 4(b) shows which of them have been correctly 


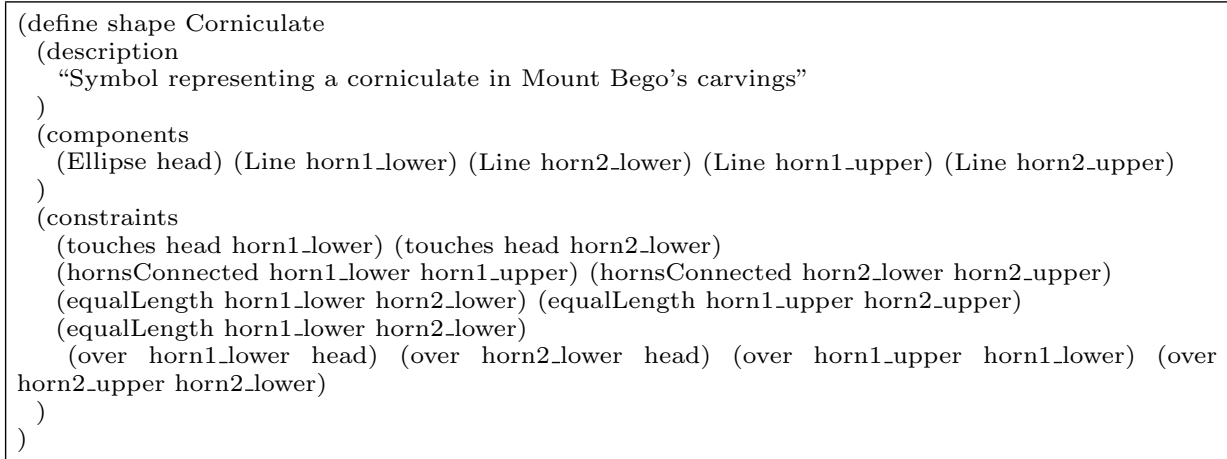

Fig. 3. LADDER description of a corniculate.

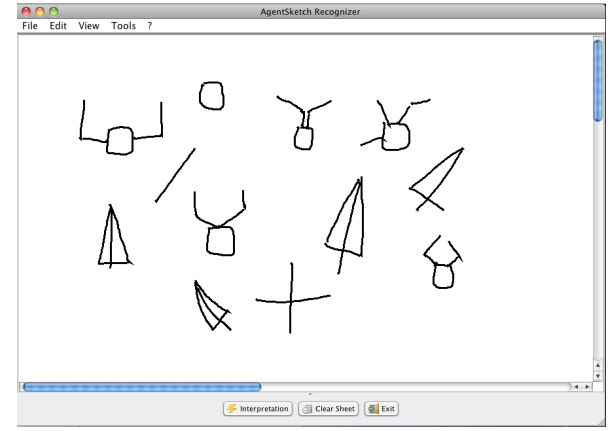

a

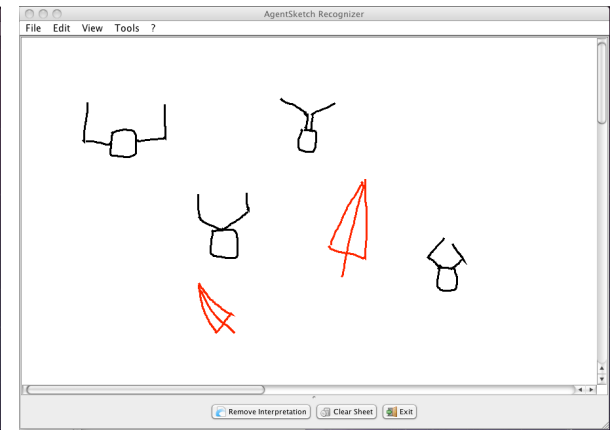

$\mathrm{b}$

Fig. 4. The user interface of the system.

recognized as corniculates (black symbols) and as daggers (red - or light gray if printed in gray-scale - symbols). All the symbols have been correctly recognized as belonging to their own category, apart from the dagger in the lower left portion of the screen. Surprisingly, despite the simplicity of their shape, correctly recognizing daggers raises some problems. Daggers that are sketched almost in the same way may be either recognized or not, depending on very small variations of the length and relative position of their constituent elements. Relaxing the constraints of the rule for recognizing daggers would eliminate this problem, but would lead to detection of false positives that, in the current setting, are completely absent. We prefer to keep the rule constraints stricter and having some weapons not recognized, rather than lowering the accuracy of the algorithm by correctly recognizing more weapons, but also having more false positives. 


\subsection{SRA for Images}

The SRA for recognizing rock carvings in images operates off-line and has been developed using an existing open source library of programming functions for real time computer vision, OpenCV (Open Source Computer Vision ${ }^{1}$ ). Among the many functions offered by OpenCV, we used those that exploit Haar-like features [14] for categorizing subsections of images based on the intensity of pixels in the region, and the AdaBoost machine learning algorithm [6] for training classifiers to recognize objects in images given positive and negative samples. Some true positives and negatives recognized by the SRA are shown in Fig. 5.
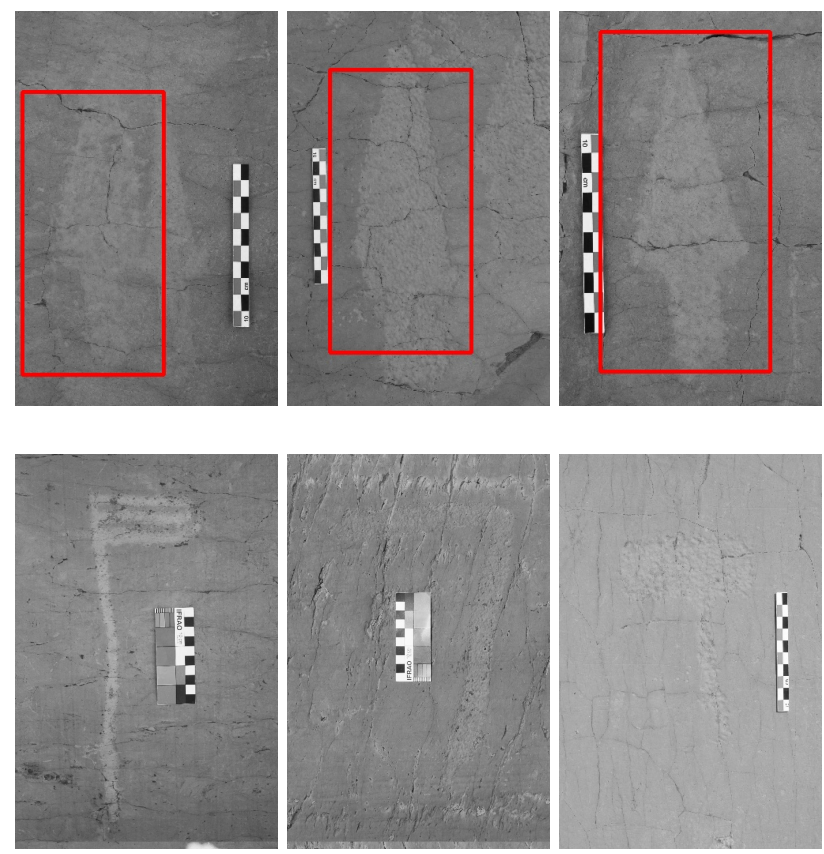

Fig. 5. True positives (top) and true negatives (bottom).

The implementation of another SRA based on SIFT [12] is under way. SIFT is a method for detecting and describing local features in images and has been recently used for automatic coin classification [16]. It could be a good choice both for hand-drawn sketches and for rock carving images, since rock engravings and ancient coins show many similarities from a computer vision viewpoint. In the coin case, SIFT could recognize the same object/element/figure in different coins even if the actual shape varies from one item to another. We are confident to reach the same good results on rock carvings with the SRA we are implementing.

\footnotetext{
${ }^{1}$ http://opencv.willowgarage.com/wiki/. Last accessed: February the 15th, 2012.
} 


\subsection{Interpretation as cooperation among holons}

Interpreting a given shape within a sketch or an image in the correct way is performed by the standalone AgentSketch (namely, AgentSketch not integrated within Indiana MAS) by exploiting contextual information local to the sketch. For example, in the domain of use case UML diagram, an oval could be interpreted either as the head of a stick man, or as a use case. If there are other strokes in the oval's context (namely, close to it) that follow the pattern of the stick man body, then the oval is more likely to be a head than a use case. In [2] we showed that exploiting such kind of contextual information improves the precision of the interpretation.

When sketches are as complex as rock carvings, however, the context to take into account to provide a correct and precise interpretation cannot be any longer limited to neighboring strokes, and cannot be hard-wired within the AgentSketch Symbol Interpretation Agent.

To make an example, in [5] a systematic analysis of meaningful associations between symbols characterizing Mount Bego's engravings have been carried out, leading to the identification of recurrent patterns such as:

- the dagger, symbol of the light, placed over a reticulate symbolizing the earth; the dagger between the horns of a corniculate (interpretation: the god of storms that fertilizes the earth by means of the rain);

- sinuous lines symbolizing the stream; the canal watering the earth and corniculates associated with sinuous lines and small corniculates; a water basin between the horns of a corniculate (interpretation: water fertilizing the earth);

- a bull below a feminine figure with open arms and legs (interpretation: the myth known as the "son-husband", a symbolic representation of the high goddess or mother goddess giving birth to the bull, who in turn fertilizes the goddess).

Hard-wiring all such patterns within AgentSketch in order to reinforce, for example, the hypothesis that a given shape is a bull if it is below a feminine shape, is not feasible: relationships among symbols are too many, too complex and too dynamic, due to the many co-existing hypotheses made by archaeologists, and to the new ones that are continuously proposed.

The solution implemented in Indiana MAS decouples the discovery and representation of such patterns among symbols from their exploitation during the sketch interpretation stage. The protocol followed by holons to boost interpretation is the following.

1. AgentText is fed with multi-lingual textual documents that it processes to perform the following operations:

(a) Categorize documents according to the Rock Carving Vocabulary, defined a priory by the domain experts at the set up of Indiana MAS; this activity is carried out by exploiting the techniques discussed in [11]. The output of the categorization activity is sent to AgentLib which stores the documents in the digital library and adds meaningful metadata to them, consistent with the categories discovered by AgentText. 
(b) Extract information about patterns like those described above; this activity is carried out by exploiting the Role Ontology Extractor [1] that we experimented in the archeological domain, as described in [13]. Discovered patterns are stored in the Rock Carving Vocabulary which is implemented as a OWL ontology [15], and hence allows us to easily define relationships among concepts in form of OWL properties that connect them (for example, concepts Dagger and Horn can be connected by the "between" property, and concepts Dagger and Reticulate by the "over" property, suitably annotated by a pointer to the document where the relationship was extracted from).

Since the success of pattern extraction heavily depends on natural language processing and word sense disambiguation, that are known to be AI-complete problems, domain experts are required to validate patterns proposed by AgentText before they are stored within the Rock Carving Vocabulary. Although not trivial, classification of documents is a more stable task than pattern extraction, and no supervision is required on it.

2. AgentSketch accesses the Rock Carving Vocabulary looking for stored patterns involving symbols that have been recognized so far, whenever it needs to disambiguate the interpretation of a sketch. Patterns that support a given interpretation, make that interpretation stronger. The strongest interpretation is proposed to the user.

This way, patterns are (semi-)automatically extracted from textual documents and represented in a declarative way. No matter what these patters are, where do they originate from, and how fast they evolve during time: AgentSketch always operates in a way that is independent from the values of the patterns themselves, and the resulting interpretation depends on the current set of patterns stored in the Rock Carving Vocabulary.

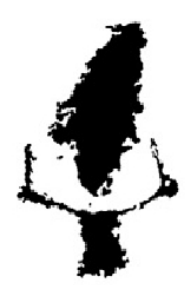

Fig. 6. Mount Bego's engravings ZIV.GII.R19C.no 12 and 13.

To make an example, let us suppose that a sketch like the one represented in Fig. 6 (Mount Bego's engravings ZIV.GII.R19C.no 12 and 13 [5]) is input to AgentSketch. The Symbol Recognizer devoted to recognizing corniculates succeeds in detecting a corniculate in the bottom part of the sketch with a high 
confidence, but the Symbol Recognizer that should recognize daggers fails in its purpose: the shape in the sketch does not meet the standard dagger shape, and the confidence that could be a dagger is below the usually acceptable threshold. On the other hand, the confidence that it is a sinuous line or a water basin, as computed by the Symbol Recognizers in charge for these two kinds of shapes, is even lower than the confidence that it is a dagger.

AgentSketch looks for patterns involving corniculates in the Rock Carving Vocabulary, and finds the following ones:

- WaterBasin between Horns belongingTo Corniculate

- Dagger between Horns belongingTo Corniculate

- Corniculates closeTo SinuousLines

These patterns make the interpretation of the unknown shape as a WaterBasin, a Dagger, or a SinuousLine feasible, but since confidence(Dagger) > confidence(WaterBasin) and confidence(Dagger) > confidence(SinuousLine), the interpretation proposed to the user is that the sketch represents a dagger between the horns of a corniculate.

Since each pattern in the Rock Carving Vocabulary contains meta-data about the document it was extracted from, the user is also shown the document as a witness for the given interpretation.

\section{Conclusions and Future Work}

The digital preservation, classification, and interpretation of rock carvings raises many scientific challenges, such as the integration of data coming from multiple sources, and the interpretation of drawings whose meaning may vary based on contextual information. The Indiana MAS project tackles such issues by exploiting intelligent agents that ensure the required degree of flexibility and autonomy in a highly dynamic and heterogeneous environment. Even if the project did not even start yet, many components of the Indiana MAS have already been developed and tested on the project's domain, and their integration in the Indiana MAS is under way. The steps moved in the rock art interpretation direction discussed in this paper, although preliminary, are very promising. The full achievement of all the project's objectives will be our main goal in the next three years.

\section{Acknowledgments}

This research is supported by the "Indiana MAS and the Digital Preservation of Rock Carvings" FIRB project funded by the Italian Ministry for Education, University and Research, under grant RBFR10PEIT.

The authors are grateful to Francesca Odone for her helpful comments on Section 3.2 . 


\section{References}

1. M. Bozzano, A. Locoro, M. Martelli, and V. Mascardi. Trattamento del linguaggio naturale tramite Prolog: un approccio promettente per generare istituzioni virtuali da testi scritti. In $C I L C ' 10,2010$. In Italian.

2. G. Casella, V. Deufemia, V. Mascardi, G. Costagliola, and M. Martelli. An agentbased framework for sketched symbol interpretation. J. Vis. Lang. Comput., 19(2):225-257, 2008.

3. G. Casella, V. Deufemia, V. Mascardi, M. Martelli, and G. Tortora. Reasoning about hand-drawn sketches: An approach based on intelligent software agents. In M. Sebillo, G. Vitiello, and G. Schaefer, editors, VISUAL 2008, volume 5188 of LNCS, pages 302-314. Springer, 2008.

4. L. Chen and K. P. Sycara. Webmate: A personal agent for browsing and searching. In Agents, pages 132-139, 1998.

5. H. de Lumley and A. Echassoux. The Rock Carvings of the Chalcolithic and Ancient Bronze Age from the Mont Bego Area. The Cosmogonic Myths of the Early Metallurgic Settlers in the Southern Alps. L'Anthropologie, 113(5P2):9691004, 2009.

6. Y. Freund and R. E. Schapire. A decision-theoretic generalization of on-line learning and an application to boosting. In P. M. B. Vitányi, editor, EuroCOLT '95, volume 904 of $L N C S$, pages 23-37. Springer, 1995.

7. E. Friedman-Hill. Jess, the rule engine for the Java platform. Sandia National Laboratories, Livermore, CA. Online at http://www. jessrules.com/. Last accessed: February the 15th, 2012.

8. C. Gerber, J. H. Siekmann, and G. Vierke. Holonic multi-agent systems. Technical Report DFKI-RR-99-03, Deutsches Forschungszentrum für Künztliche Inteligenz GmbH, Postfach 20 80, 67608 Kaiserslautern, FRG, 1999.

9. T. Hammond and R. Davis. LADDER, a sketching language for user interface developers. Computers \&5 Graphics, 29(4):518-532, 2005.

10. H. Lieberman. Letizia: An agent that assists web browsing. In 14th Int. Joint Conference on Artificial Intelligence, pages 924-929. Morgan-Kaufmann, 1995.

11. A. Locoro, D. Grignani, and V. Mascardi. MANENT: An infrastructure for integrating, structuring and searching digital libraries. In M. Biba and F. Xhafa, editors, Learning Structure and Schemas from Documents, volume 375 of Studies in Computational Intelligence, pages 315-341. Springer, 2011.

12. D. G. Lowe. Object recognition from local scale-invariant features. In International Conference on Computer Vision, pages 1150-1157. IEEE Computer Society, 1999.

13. L. Papaleo, G. Quercini, V. Mascardi, M. Ancona, A. Traverso, and H. D. Lumley. Agents and ontologies for understanding and preserving the rock art of mount bego. In J. Filipe and A. L. N. Fred, editors, ICAART 2011, Vol. 2 - Agents, pages 288-295. SciTePress, 2011.

14. P. A. Viola and M. J. Jones. Rapid object detection using a boosted cascade of simple features. In CVPR 2001, pages 511-518. IEEE Computer Society, 2001.

15. W3C. OWL 2 Web Ontology Language Document Overview - W3C Recommendation 27 October 2009, 2009.

16. S. Zambanini and M. Kampel. Automatic coin classification by image matching. In F. Niccolucci, M. Dellepiane, S. P. Serna, H. E. Rushmeier, and L. J. V. Gool, editors, VAST 2011, pages 65-72. Eurographics Association, 2011. 